\title{
3-D Tracking in a Miniature Time Projection Chamber
}

\author{
S. E. Vahsen*, M. T. Hedges, I. Jaegle, S. J. Ross, I. S. Seong, T. N. Thorpe, J. Yamaoka
}

University of Hawaii, 2505 Correa Road, Honolulu, HI 96822, USA

J. A. Kadyk, M. Garcia-Sciveres

Lawrence Berkeley National Laboratory, 1 Cyclotron Road, Berkeley, CA 94720, USA

\begin{abstract}
The three-dimensional (3-D) detection of millimeter-scale ionization trails is of interest for detecting nuclear recoils in directional fast neutron detectors and in direction-sensitive searches for weakly interacting massive particles (WIMPs), which may constitute the Dark Matter of the universe. We report on performance characterization of a miniature gas target Time Projection Chamber (TPC) where the drift charge is avalanche-multiplied with Gas Electron Multipliers (GEMs) and detected with the ATLAS FEI3 Pixel Application Specific Integrated Circuit (ASIC). We report on measurements of gain, gain resolution, point resolution, diffusion, angular resolution, and energy resolution with low-energy x-rays, cosmic rays, and alpha particles, using the gases $\mathrm{Ar}: \mathrm{CO}_{2}(70: 30)$ and $\mathrm{He}: \mathrm{CO}_{2}(70: 30)$ at atmospheric pressure. We discuss the implications for future, larger directional neutron and Dark Matter detectors. With an eye to designing and selecting components for these, we generalize our results into analytical expressions for detector performance whenever possible. We conclude by demonstrating the 3-D directional detection of a fast neutron source.
\end{abstract}

Keywords: TPC, GEM, pixel, directional, neutron, dark matter

\section{Introduction}

Time Projection Chambers [1] with charge readout via micro-pattern gaseous detectors are the digital analog to bubble chambers, allowing 3-D reconstruction of ionization with many space points and great precision. Over the last decade, a number of studies have demonstrated impressive performance when reconstructing ionizing primary particles with such detectors $[2,3,4]$. Our group is investigating $[6,7,8,9,10]$ the detection of nuclear recoils resulting from the scattering of neutral primary particles, such as neutrons and, potentially, WIMPs. Neutron detectors with improved directional sensitivity are likely to find applications in particle physics, nuclear physics, homeland security, and neutron imaging. Directional searches for WIMP Dark Matter are sensitive to a unique signature, a 24hour directional oscillation of the WIMP recoil distribution, due to the rotation of the earth [11]. Observation of this signature would constitute a convincing detection of WIMPs by demonstrating the galactic origin of the signal, and may be required to distinguish WIMP scattering from coherent neutrino scattering [12]. A number of technological approaches are being explored $[13,14,15,16,17,18,19,20,21]$. An ideal directional WIMP detector, capable of excluding the isotropy of nuclear recoils in galactic coordinates with order ten signal events, would track nuclear recoils in 3-D, with low energy threshold, and with the ability to detect which of the two possible directions along an

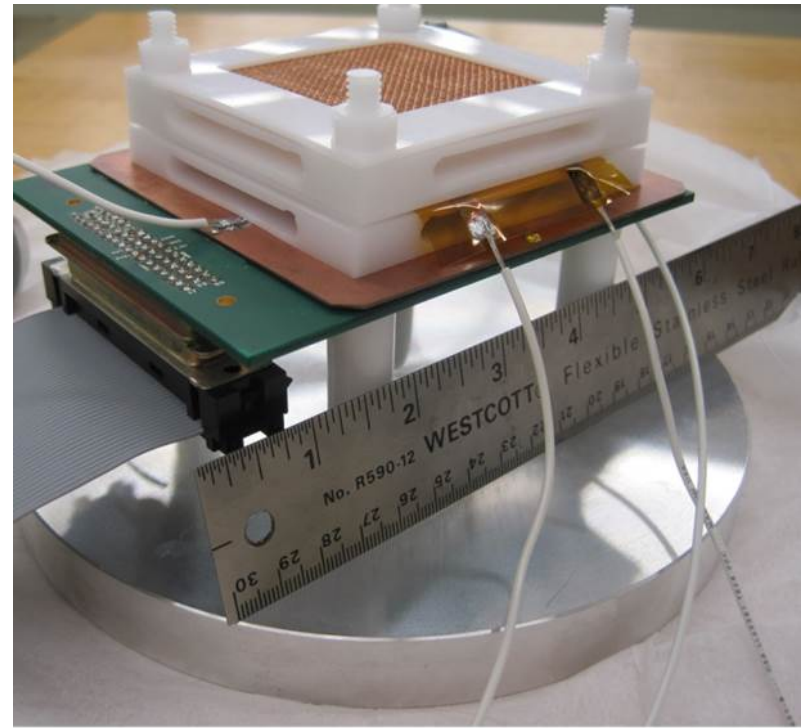

Figure 1: $\mathrm{D}^{3}$-Micro prototype in the original configuration used for most studies presented here. The sensitive volume consists of a 9.2-mm vertical drift gap between the copper cathode (mesh visible on the top of the detector) and the top GEM (foil protruding on the right).

${ }^{*}$ Corresponding author. Tel.: +1 8089562985 .

Email address: sevahsen@hawaii.edu (S. E. Vahsen) 
observed track the recoiling nucleus moved [22]. The latter is also sometimes referred to as vector tracking, sense recognition, or head/tail recognition. The technology under study is a candidate for building such a detector. One obvious challenge for gas-based WIMP searches is low target mass per unit volume. However, the proper metric for comparing technologies is sensitivity per unit of cost. For high-resolution gas TPCs, the cost drivers are typically the readout plane and electronics. If the cost of these can be minimized, for instance by focusing the drift charge onto the detection plane [7], large gas TPCs will be more competitive.

We report here on the performance of a miniature prototype, $\mathrm{D}^{3}$-Micro (Directional Dark Matter Detector - Micro), constructed at the University of Hawaii in 2010. In that detector the TPC drift charge is multiplied with a double layer of Gas Electron Multipliers (GEMs) [23] and detected with the ATLAS FEI3 Pixel Application Specific Integrated Circuit (ASIC) [24]. The high double GEM gain (of order $10^{4}$ ), low pixel threshold (typically 2000-4000 $\mathrm{e}^{-}$), and low pixel noise (typically 100$200 \mathrm{e}^{-}$) result in several attractive features, such as stable operation with single electron efficiency near unity, self-triggered readout, and negligible rates of noise hits. In practical terms, this means that at high gain, essentially all primary ionization can be detected, so that the energy threshold is equal to the work function of the gas, typically about $30 \mathrm{eV}$. Therefore one can expect a large number of hits even for $\mathrm{keV}$-scale tracks. It seems likely that these outstanding capabilities will enable reconstruction of tracks with the lowest energy threshold possible in any detector of ionization. Due to the self-triggering capability of the pixel chip, the detector produces no data in the absence of ionization in the drift gap, greatly reducing the requirements on DAQ electronics. This is important in the context of scaling to larger detectors.

All measurements presented here were carried out at atmospheric pressure (760 Torr). Initial measurements employed Ar: $\mathrm{CO}_{2}$ (70:30), which is a commonly used detector gas, and allows a comparison with work by others [25]. Later measurements were performed with $\mathrm{He}: \mathrm{CO}_{2}$ (70:30), which is more suitable for reconstructing fast neutron recoils. Helium is a good neutron target, since up to $64 \%$ of the neutron energy can be transfered to a helium nucleus. Helium is also a good detection medium, since the low electron density results in small specific ionization, yielding longer recoil tracks. The $\mathrm{CO}_{2}$ component improves detector performance and stability by reducing diffusion, by re-absorbing UV-photons emitted during the avalanche process (commonly referred to as quenching), and by raising the electric field strength threshold for sparking.

\section{Detector and Principle of Operation}

The $\mathrm{D}^{3}$-Micro prototype consists of a Delrin (acetal) support structure, visible as white parts in Figs. 1 and 2, on which the different electrical components are mounted. The support structure resides inside a 25-liter stainless steel test vessel. Much of the detector design, shown schematically in Fig. 3, is based on a previous prototype constructed at LBNL [4]. The sensitive volume of the detector consists of a drift gap situated be-

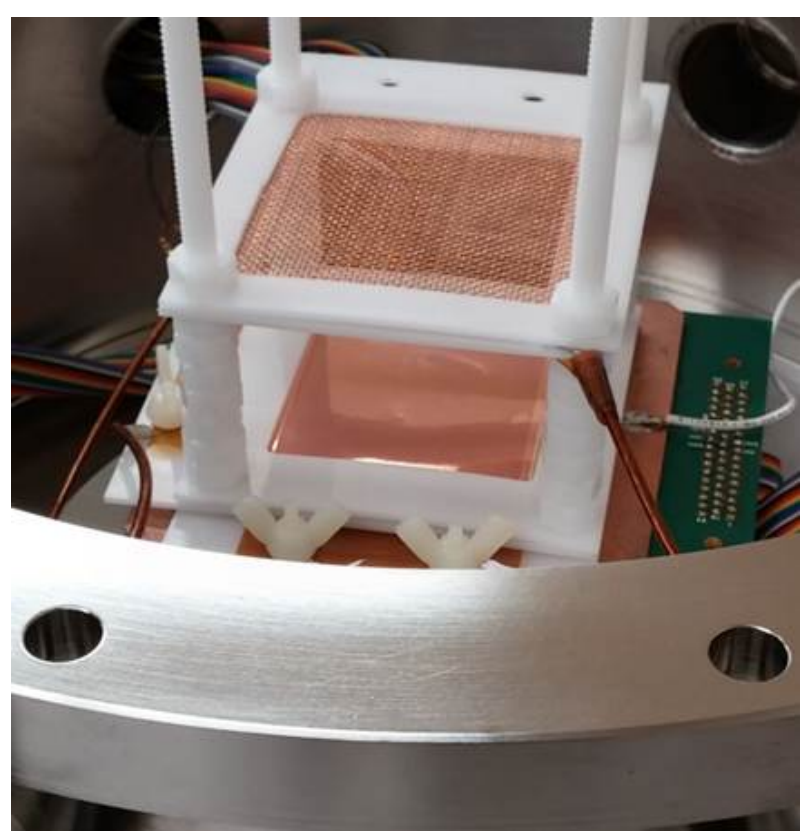

Figure 2: $\mathrm{D}^{3}$-Micro prototype in the test vessel, after the drift gap was increased to $45 \mathrm{~mm}$, and the thickness of nearby Delrin (acetal) parts was reduced. These modifications were crucial for achieving a significant directional neutron signal.

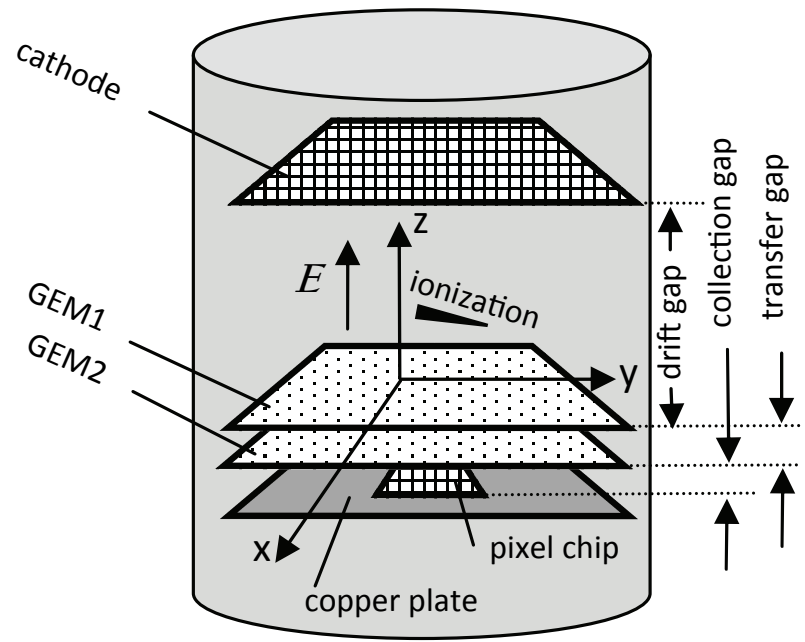

Figure 3: Schematic representation of the $\mathrm{D}^{3}$-Micro prototype and definition of coordinate system. The origin is chosen to that $x=0, y=0$ coincides with one corner of the pixel chip and $z=0$ is at the bottom of the drift gap. The $x$ and $y$ axes are parallel to the 7.2- $\mathrm{mm}$ and $8.0-\mathrm{mm}$ sides of the pixel chip, respectively. The $z$ axis points in the direction of the drift field, i.e. opposite to the direction of electron drift. The rectangular pixels measure $400 \mu \mathrm{m} \times 50 \mu \mathrm{m}$ in $x \times y$. For fitted tracks, we use the spherical coordinate convention most common in physics: the polar angle, $\theta$, is the angle between the $z$-axis and the track's direction vector. The azimuthal angle, $\phi$, is the angle between the $x$-axis and the projection of the direction vector onto the $x$ - $y$ plane. 
tween a copper mesh and the upper surface of GEM1. For most ${ }_{138}$ measurements the drift gap was $9.2 \mathrm{~mm}$. For the demonstration of neutron detection, higher detection efficiency was re- ${ }^{139}$ quired, and the drift gap was increased to $45 \mathrm{~mm}$, as shown in ${ }^{140}$ Fig. 2. Ionizing radiation produces free electrons in this gap. ${ }^{141}$ These electrons then drift in a uniform electric field to a dou- ${ }^{142}$ ble GEM layer, where the electrons are avalanche multiplied, ${ }^{143}$ and finally the resulting avalanche charge is detected with an ${ }^{144}$ ATLAS FE-I3 pixel chip [24] operating in self-trigger mode ${ }^{145}$ (described below) and sampling at $40 \mathrm{MHz}$. The GEMs used ${ }^{146}$ are the standard CERN design with an active area of $5 \times 5 \mathrm{~cm}^{147}$ and $140 \mu \mathrm{m}$ hole spacing [23]. The transfer gap, defined as ${ }^{148}$ the distance between the lower surface of GEM1 and the upper ${ }^{149}$ surface of GEM2, is $2.8 \mathrm{~mm}$. The collection gap, defined as ${ }^{150}$ the distance between the bottom surface of GEM2 and the up- ${ }^{151}$ per surface of the pixel chip, is $2.2 \mathrm{~mm}$. The pixel chip has an ${ }^{152}$ active area of $7.2 \times 8.0 \mathrm{~mm}^{2}$, divided into 2880 pixels. Each in- ${ }^{153}$ dividual $50 \times 400 \mu \mathrm{m}^{2}$ pixel contains an integrating amplifier, $\mathrm{a}^{154}$ discriminator, a shaper, and associated digital controls. A pix- ${ }^{155}$ elized metal layer was deposited [4] onto the chip to increase ${ }^{156}$ the charge collection efficiency, but this led to a non-uniform ${ }^{157}$ efficiency, see section 3 .

When charge is detected in the chip (at least one pixel detects ${ }^{159}$ charge above threshold), the self-trigger results in the output of ${ }^{160}$ a zero-suppressed digital serial stream that encodes the 2-D po- ${ }^{161}$ sition, arrival time, and amount of charge collected, for each ${ }^{162}$ pixel above threshold within the next sixteen cycles of $25 \mathrm{~ns}^{163}$ each. The charge collected in each pixel is deduced from the ${ }^{164}$ time above threshold (ToT), which is measured with 7-bit pre- ${ }^{165}$ cision. By using the known drift velocity in the drift gap, the ${ }^{166}$ timing information is converted into a (relative) third spatial ${ }^{167}$ coordinate, so that a 3-D image of ionization in the drift gap is ${ }^{168}$ obtained, as shown in Figs. 10, 12, and 17. The pixel chip is ${ }^{169}$ glued to a circuit board and electrically connected with wire- ${ }^{170}$ bonds, which are shielded against the electric field with a small ${ }^{171}$ metal overhang, as described in [4]. In addition to the digital ${ }^{172}$ charge readout via the pixel chip, the area surrounding the chip ${ }^{173}$ is covered with a copper plate which is connected via a capac- ${ }^{174}$ itor to an Endicott eV-5093 charge sensitive preamplifier. The ${ }^{175}$ amplifier output is fed through a Canberra AFT 2025 shaping $^{176}$ amplifier into an Ortec EASY-MCA operating in pulseheight ${ }^{177}$ analyzer (PHA) mode. The PHA is used to measure gain and ${ }^{178}$ gain resolution of the double GEM, but is not used when tracks ${ }^{179}$ are recorded with the pixel chip.

For the studies described here, the test vessel was typically ${ }^{181}$ pumped down to $10^{-4}$ Torr, and then filled with the target gas ${ }^{182}$ under study. To ensure good gas purity, we usually performed ${ }^{183}$ multiple such pump-and-fill sequences before data taking, re- ${ }^{184}$ peated the gain studies with and without gas flow during detec- ${ }^{185}$ tor operation, and checked for long term gain stability, which ${ }^{186}$ was better than $2 \%$ over several weeks. For studies with x-rays ${ }^{187}$ and alpha-particles, radioactive sources were placed inside the vessel. For neutron detection studies, we placed the source out ${ }_{188}$ side the vessel. We adjusted the double GEM gain and drift field depending on the energy scale and requirements of each ${ }^{189}$ particular study. The different settings are summarized in Table ${ }_{190}$ 1.

\section{Comment on Inefficient Pixels}

The FE-I3 pixel chip, as used in ATLAS, has a nonconductive surface, except for a $12 \mu \mathrm{m}$-wide octagonal metal bond pad per $400 \times 50 \mu \mathrm{m}^{2}$ pixel. In order to increase the fractional surface area sensitive to charge, we evaporated a pixelized metal layer onto the chip [4]. However, imperfections in this process led to a non-uniform detector response, with a different pattern for each chip.

For the particular chip used here, despite good GEM gain uniformity (better than 10\%, measured with FE-55 and a PHA) and excellent pixel chip electronics uniformity (better than $3 \%$, measured with a chip internal test pulser), pixels in every second $400-\mu \mathrm{m}$ pixel column collect less charge. With all pixels tuned to a threshold of 2200 electrons, the inefficient pixels have a lower ToT response to charge in the drift gap, and effectively behave as if the threshold were higher than the tuned value. The resulting hit-count inefficiency is charge-density dependent; for alpha particles, averaged over the chip, it is $40 \%$. To improve the chip uniformity, for each measurement performed here, we left the threshold of inefficient pixels at 2200 electrons, but raised the threshold of all efficient pixels until we observed a more uniform hit count across the chip, i.e. we deliberately trade some sensitivity to remove the large non-uniformity due to the metalization. The threshold in the efficient pixels is thus the effective threshold of the pixel chip, listed for each measurement in Table 1. While this calibrated out the largest non-uniformity, there is significant residual nonuniformity in response, both in efficiency and in TOT calibration, see Fig. 4. We have since resolved the issue with an improved metalization process, and already built next-generation detectors with a uniform efficiency and ToT response.

Given these development, it did not seem worthwhile to further pursue a perfectly uniform response in the present detector. Hence we report here mainly on measurements that do not use the ToT and which are intended to be robust against pixel-level inefficiencies. If there is a weak sensitivity, we expect that our results are conservative, in that they should improve with ToT weighting or a fully efficient, uniform metal layer. Specifically, the gain resolution measurements do not use the pixel chip, hence are unaffected by non-uniform pixel response. The cosmic ray point resolution measurements do not use ToT weighting, and use pixel-level residuals (as opposed to cluster-level), to avoid bias from inefficient pixels. The alpha track segment fits do not use ToT weighting. In addition, to provide a result that factors out the pixel-level inefficiencies, we parameterized the alpha segment angular resolution in terms of the number of pixels detected. Measurements of particle ID (via specific ionization) and of energy depend fully on the ToT measurement. Hence we only present one such measurement (the energy resolution for alpha track segments) and discuss its limitations.

\section{Measurements of Gain and Gain Resolution}

\subsection{X-rays}

We measure the effective double-GEM gain and its resolution by placing an uncollimated $\mathrm{Fe}-55$ 5.9-keV x-ray source on 


\begin{tabular}{|l|c|c|c|c|c|c|}
\hline $\begin{array}{l}\text { Source of } \\
\text { ionization }\end{array}$ & $\begin{array}{c}\text { effective } \\
\text { threshold }\left(e^{-}\right)\end{array}$ & $V_{\text {GEMS }}$ & $\begin{array}{c}\text { effective gain } \\
\left(10^{3}\right)\end{array}$ & $\begin{array}{c}E_{\text {drift }} \\
(\mathrm{kV} / \mathrm{cm})\end{array}$ & $\begin{array}{c}E_{\text {transfer }} \\
(\mathrm{kV} / \mathrm{cm})\end{array}$ & $\begin{array}{c}E_{\text {collection }} \\
(\mathrm{kV} / \mathrm{cm})\end{array}$ \\
\hline cosmic rays & 4000 & 983 & 41 & 1.2 & 3.7 & 2.7 \\
alpha particles & 10000 & 833 & 3.2 & 0.84 & 2.3 & 3.1 \\
neutron recoils & 8000 & 806 & 2.0 & 0.64 & 2.2 & 3.0 \\
\hline
\end{tabular}

Table 1: Effective pixel threshold, effective gain, and electric field settings used for the different studies presented. $V_{G E M S}$ is the sum of the two, nearly equal, voltages across GEM1 and GEM2. .
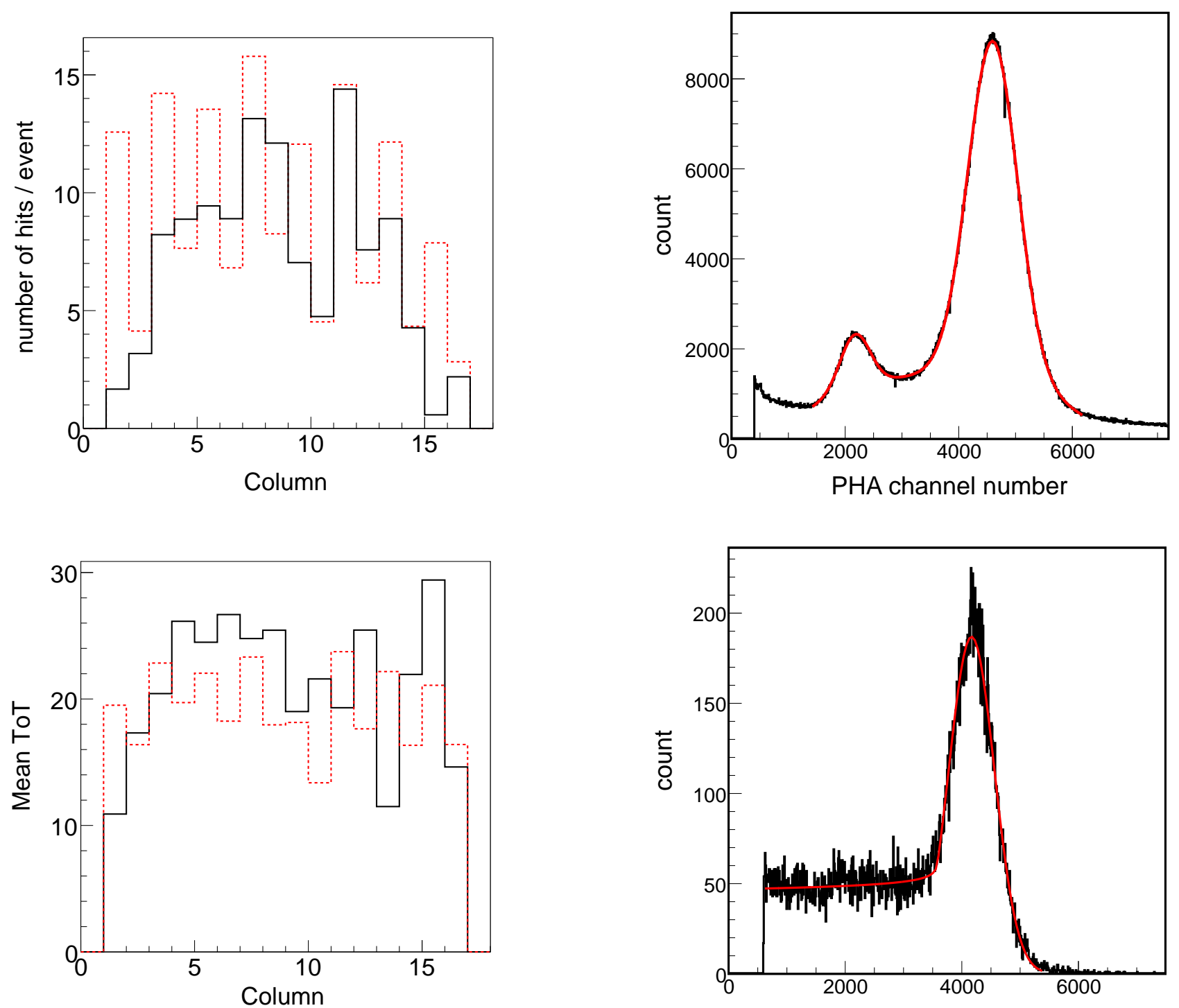

Figure 4: Non-uniformity of the pixel chip, due to imperfect metalization, measured with alpha particle events. Dashed plots: all pixels were tuned to a threshold of 2200 electrons. Solid plots: pixels in every second column were tuned to a threshold of 10000 electrons. Top: Mean column-occupancy versus column number. Bottom: Mean ToT per pixel versus column number.

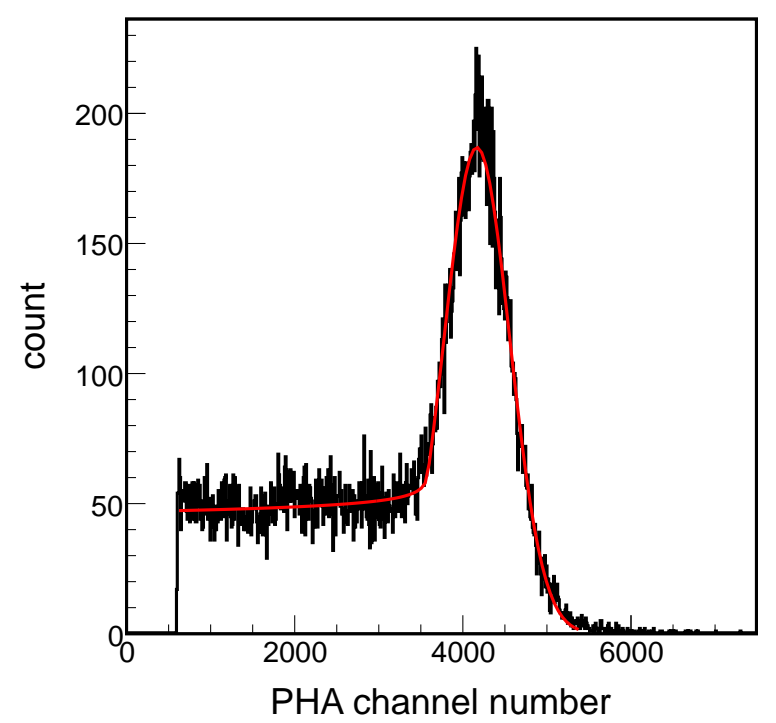

Figure 5: Pulseheight spectra recorded with an Fe-55 x-ray source using $\mathrm{ArCO}_{2}$ (upper) and $\mathrm{HeCO}_{2}$ (lower) gas and a double-GEM gain of $4 \times 10^{4}$. The black points show experimental data. The smooth curves (red in online version) are the result of fits to the data, as described in the text. 


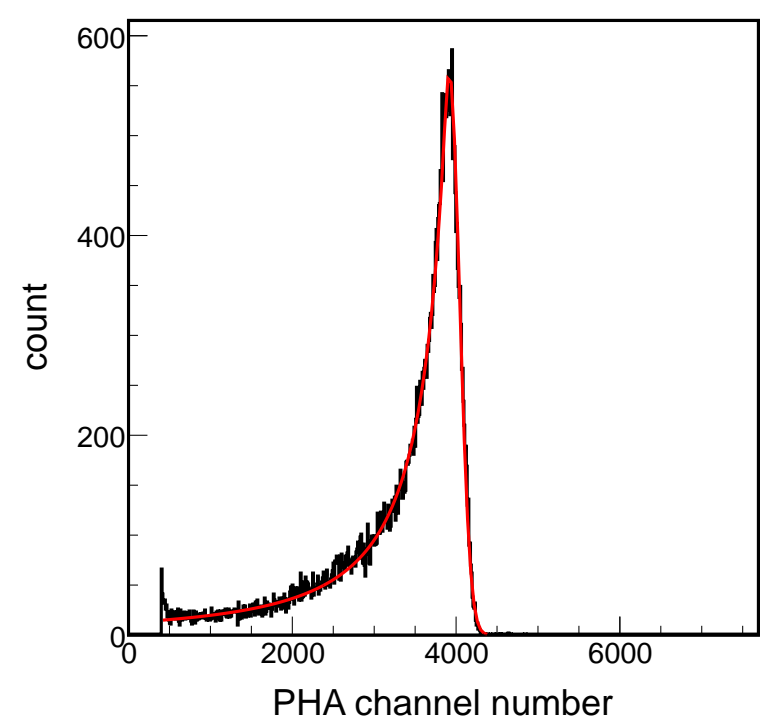

Figure 6: Pulseheight spectrum recorded with a Po-210 alpha source in $\mathrm{ArCO}_{2}$ gas at a double-GEM gain of approximately 45 . The black points show experimental data. The smooth curve (red online) is the result of a fit to the data, as described in the text.

top of the cathode mesh so that the avalanche charge is incident on the copper pad, and observe the resulting pulse height spec- ${ }^{240}$ tra in the PHA. Two of the many spectra measured are shown in $^{241}$ Fig. 5. We perform a $\chi^{2}$-minimization to the spectra, where the ${ }^{242}$ $\mathrm{ArCO}_{2} \mathrm{x}$-ray conversion signal is modeled by the sum of $\mathrm{two}^{243}$ Gaussians (the argon main peak and escape peak). We perform ${ }^{244}$ each fit twice, once with the noise background in the PHA spec- ${ }^{245}$ trum modeled by a polynomial of degree two, and once with the ${ }^{246}$ noise modeled by a power-law function. We take the mean $\mathrm{fit}^{247}$ parameters obtained with the two background models as our re-248 sult, and the differences between results with the two models, in quadrature with fitting uncertainties, as systematic uncertainties. For $\mathrm{HeCO}_{2}$ spectra we model the signal with a Crystal Ball function [26], i.e. a Gaussian peak with a power-law low-end tail below an adjustable threshold, and obtain a good fit to the relevant part of the spectrum without including any additional background component. For both gases, the mean of the Gaus-249 sian is used to calculate a gain value, and the sigma of the Gaus-250 sian is used to calculate the gain resolution at that gain, as fol-251 lows: We convert PHA channel numbers into detected charge 252 values using the measured response of the PHA and amplifier ${ }_{253}$ chain. The latter was measured to be $0.91 \pm 0.13 \mathrm{~V} / \mathrm{pC}$, using an ${ }_{254}$ injection capacitor and voltage pulse generator. The quoted un-255 certainty is systematic, limited by our measurement of the small 256 injection capacitance. As a result, all effective gain measure-257 ments presented have a common, $14 \%$ systematic uncertainty, which we do not include in error bars in any figures. We assume that the x-ray conversion yields 210 electrons for the main peak in $\mathrm{ArCO}_{2}$, and 172 electrons for the peak in $\mathrm{HeCO}_{2}$ [27]. The ratio of detected charge to the $\mathrm{x}$-ray conversion yield is taken to be the effective gain.

\subsection{Alpha Particles}

Since the gain resolution improves with increasing number of primary electrons, we also performed $\mathrm{ArCO}_{2}$ gain and resolution measurements with a Po-210 5.3-MeV alpha particle source. The source was aimed horizontally so that the alpha particles would enter and stop in the drift gap above the copper pad that feeds the PHA. In this configuration we expect $4 \pm 1.3 \mathrm{MeV}$ of primary ionization to contribute to the PHA signal. The large uncertainty on this initial energy stems from an uncertainty in the exact position of the source. As can be seen in Fig. 6, the resulting PHA spectra are much narrower than those at lower energies. We fit the PHA spectra with a Crystal Ball function, and extract the gain and its resolution as described above for $\mathrm{x}$-rays. Note that this implies that the narrow upper tail of the pulseheight peak is used to measure the gain resolution.

\subsection{Dependence on GEM Voltage, Gas Type, and Energy}

We were able to operate the detector stably and for weeks at a time with double GEM gains as high as $4 \times 10^{4}$. Figure 7 summarizes the measured gain versus double-GEM voltage, where each gain value was obtained from a fit to a PHA spectrum as discussed above. The dependence of gain on GEM voltage is well described by the function

$$
G=10^{\frac{V_{\mathrm{GEM}}-V_{1}}{V_{2}}}
$$

where $G$ is the gain, $V_{\mathrm{GEM}}$ is the sum of the voltages across the two GEMs, and $V_{1}$ and $V_{2}$ are free parameters that we extract with a $\chi^{2}$ fit, see Table 2 . These parameters agree at the $20 \%$-level with measurements of our previous prototype [4] and measurements by other groups [25]. When extrapolated to the same GEM voltages, the effective gain measured with the Po210 alpha source is slightly lower than that measured with the Fe-55 x-ray source. This may be due to an increased loss of ionization to recombination in the case of alpha particles.

Figure 8 summarizes the measured gain resolution versus effective gain, where each resolution and gain value was obtained from a fit to a PHA spectrum as discussed above. The resolution versus gain is well described by a function of form

$$
\sigma_{G} / G=\sqrt{(a / G)^{2}+b^{2}}
$$

where $\mathrm{G}$ is the gain, $a$ a term due to noise fluctuations, and $b$ is the asymptotic detector resolution at high gain. Fitting this function to the data yields the parameters shown in Table 2. The gain resolution at $5.9 \mathrm{keV}$ becomes asymptotic (at high gain, where PHA noise becomes negligible) to $\approx 9 \%$, typical for gas detectors. The measured resolution for $\mathrm{MeV}$ signals is as low as $3.4 \%$, with the fitted function extrapolating to an asymptotic value of $2 \%$ at higher gain, i.e. approaching the excellent energy resolution of solid state detectors.

Since three of the resolution measurements were performed in $\mathrm{ArCO}_{2}$, but at different energies, we can obtain the dependence of the asymptotic (high-gain) detector resolution versus energy, as shown in Fig. 9. The three points are well described by the function

$$
\sigma_{G} / G=\sqrt{d^{2}+c^{2} / E},
$$




\begin{tabular}{|l|l|l|l|l|l|}
\hline gas mixture & ionization energy & $V_{1}$ & $V_{2}$ & $\mathrm{a}$ & $\mathrm{b}$ \\
\hline $\mathrm{ArCO}_{2}$ & $5.9 \mathrm{keV}$ & $434 \pm 9$ & $116 \pm 2$ & $(7.8 \pm 2.2) \times 10^{4}$ & $(9.4 \pm 0.6) \%$ \\
$\mathrm{ArCO}_{2}$ & $2.9 \mathrm{keV}$ & $434 \pm 18$ & $116 \pm 4$ & $(1.8 \pm 0.7) \times 10^{5}$ & $(13 \pm 4.2) \%$ \\
$\mathrm{ArCO}_{2}$ & $4.0 \mathrm{MeV}$ & $427 \pm 3$ & $130 \pm 2$ & $120.3 \pm 0.7$ & $(1.98 \pm 0.06) \%$ \\
$\mathrm{HeCO}_{2}$ & $5.9 \mathrm{keV}$ & $356 \pm 7$ & $136 \pm 2$ & $(1.375 \pm 0.003) \times 10^{5}$ & $(8.79 \pm .02) \%$ \\
\hline
\end{tabular}

Table 2: Parameters characterizing the double GEM gain and its resolution, defined by equations 1 and 2 , and with the following physical interpretations: $V_{1}$ is the double-GEM voltage that gives a gain of unity. $V_{2}$ is the double-GEM voltage change required to increase the gain by a factor of 10 . The parameter $a$ is the gain above which the gain resolution becomes asymptotic, and $b$ is the asymptotic gain resolution at high gain.
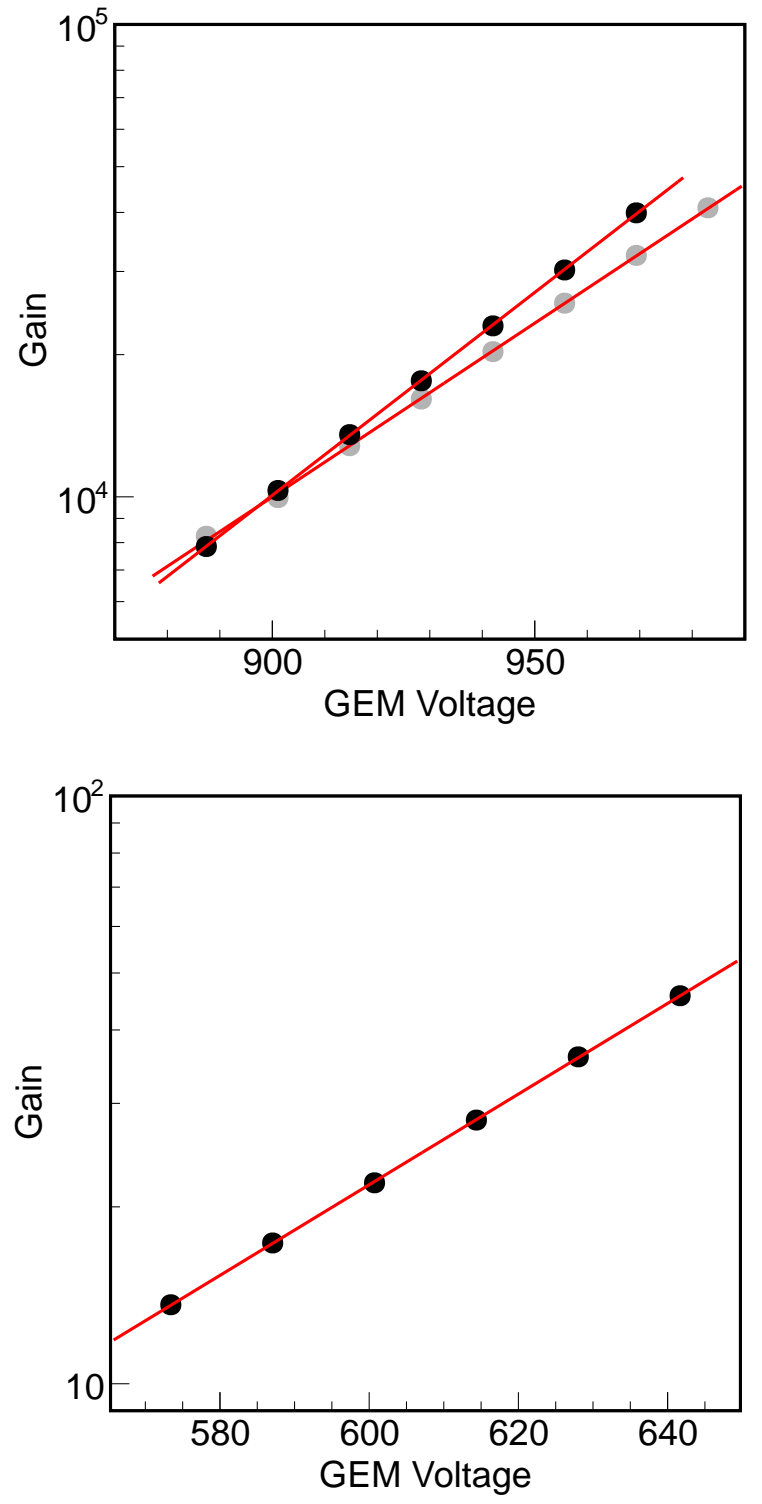

Figure 7: Effective gain of double GEM layer versus sum of GEM voltages. Top: measured for high GEM voltages with 5.9-keV x-rays in $\mathrm{ArCO}_{2}$ gas (black points) and $\mathrm{HeCO}_{2}$ gas (gray points). Bottom: measured for lower GEM voltages with 4-MeV alpha particles in $\mathrm{ArCO}_{2}$ gas. The straight lines show fits of equation 1 to the data.
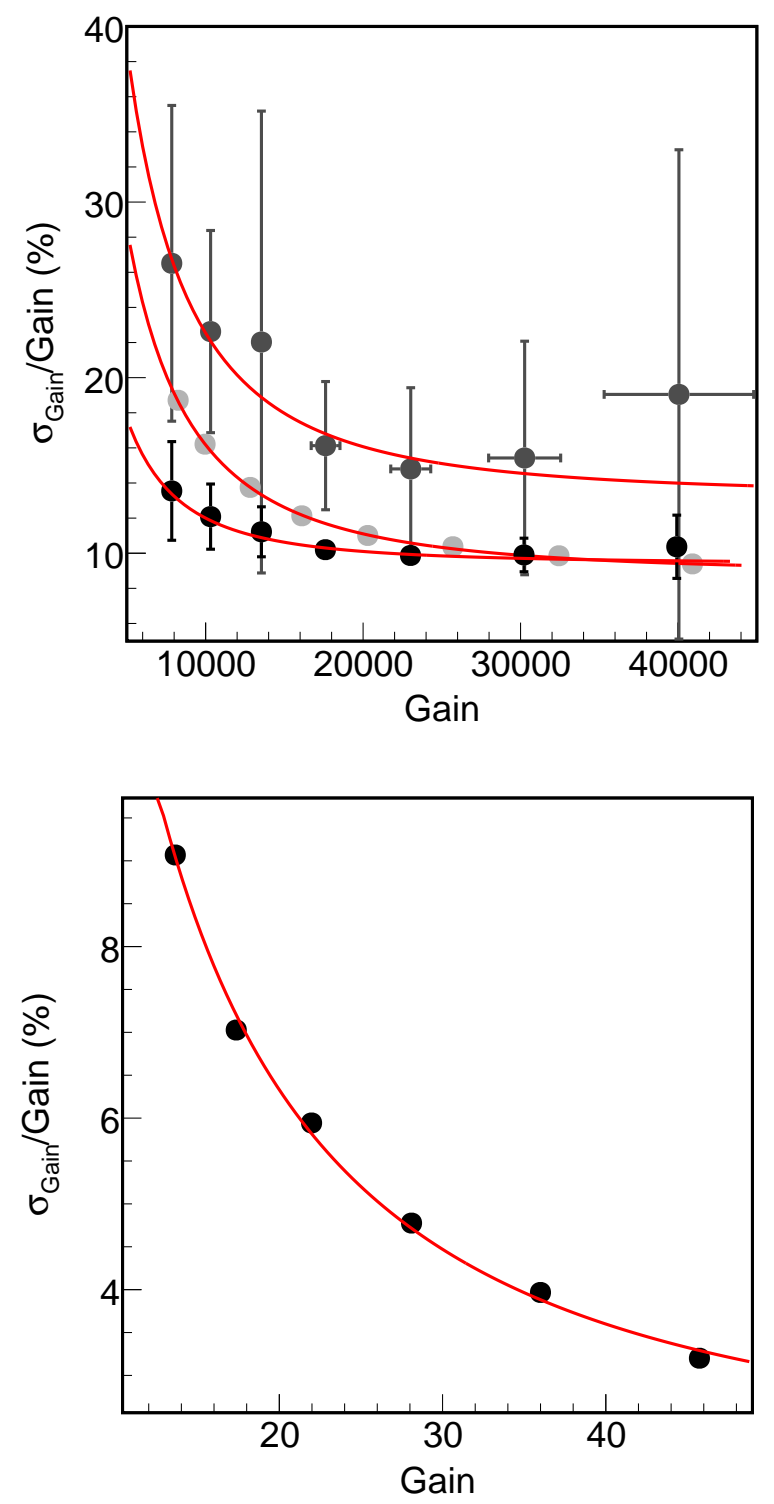

Figure 8: Gain resolution versus effective gain of double GEM. Top: measured for high GEM voltages with $\mathrm{Fe}-55 \mathrm{x}$-rays in $\mathrm{ArCO}_{2}$ gas (black points: $5.9 \mathrm{keV}$, dark gray points: $2.9 \mathrm{keV}$ ) and $\mathrm{HeCO}_{2}$ gas (light gray points, $5.9 \mathrm{keV}$ ). Bottom: measured for low GEM voltages with Po-210 alpha particles in $\mathrm{ArCO}_{2}$ gas. The curves show fits of equation 2 to the data. 


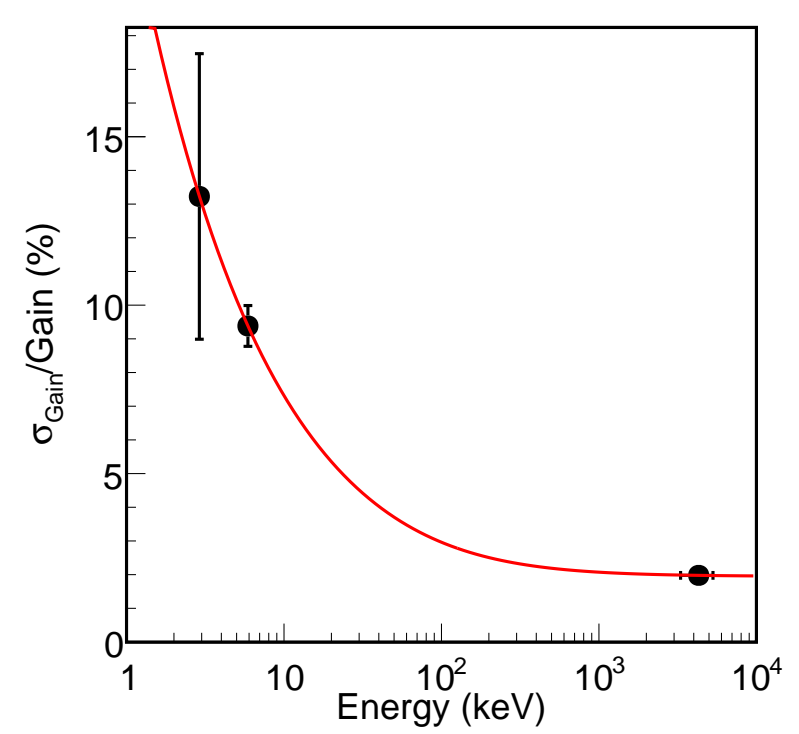

Figure 9: Asymptotic (high-gain) gain resolution versus ionization energy for $\mathrm{ArCO}_{2}$ gas. See text for discussion.

with $d=(1.94 \pm 0.07) \%$, and $c=(22.3 \pm 1.5) \% \times \sqrt{k e V}$, where $\mathrm{G}$ is the gain, $d$ is the effective gain stability of the detector and the measurement system (e.g. limited by the stability of the GEM high voltage supply, shaping amplifier, and PHA), while $c / \sqrt{E}$ is the fundamental gain resolution of the technique, determined by statistical fluctuations in the initial ionization statistics and in the avalanche process. These two statistical effects both average out as the number of primary electrons increases, which gives rise to the factor $1 / \sqrt{E}$ multiplying the $c$ term. It can be shown [28] that $c^{2}=W \times\left(F+b^{\prime}\right)$, where $W$ is the work function of the gas, $F$ is the Fano factor describing the fluctuations in primary ionization statistics [29], and $b^{\prime}$ is the Polya distribution parameter describing the variation in the avalanche gain [30]. Using our measured value for $c, W=28.05 \mathrm{eV} /$ electron and the Fano factor $F=0.23$ for gaseous Argon [31], we obtain $b^{\prime}=1.54$ for the combined double GEM layer, or $b=0.77$ per GEM.

Figure 9 leads to interesting conclusions: For the lowest energy measured, $2.9 \mathrm{keV}$, the gain resolution is of order $15 \%$, likely sufficient to provide head/tail discrimination for $\mathrm{keV}$ scale nuclear recoils, such as might be expected for low mass $(\approx 10-\mathrm{GeV})$ WIMPs. Since the resolution in the keV range is limited by the primary ionization statistics in the gas and the avalanche gain fluctuations in the GEMs, improving the detector electronics would not improve energy resolution in this energy region. Electron counting [32], however, may reduce or theoretically even fully eliminate the component of energy reso- ${ }^{306}$ lution due to gain fluctuations, and thus lead to improved energy 307 resolution in the $\mathrm{keV}$ regime in future detectors. At $\mathrm{MeV}$ scale 308 energies, of interest for fast-neutron spectroscopy, the numbersog of primary electrons is so large that statistical fluctuations in ${ }_{310}$ ionization statistics and avalanche gain become lesser effects, 311 and instead the detector and measurement system stability cur-312 rently limit the gain resolution measured. Hence improved ${ }_{313}$ pixel, shaper, and PHA electronics could lead to improved gain ${ }_{314}$ and energy resolution in this regime.

The resolution measurements are also sensitive to gain uniformity. For example, the uncollimated Fe-55 disk source used illuminates a GEM region somewhat larger than the pixel chip. While the resolution measurements presented here where all performed with the source illuminating the copper pad which surrounds the pixel chip, we also performed gain measurements in the particular GEM location below which the chip was later installed, before the detector was fully assembled. The resolution measured was comparable to the measurements presented here, meaning that GEM gain uniformity over the area of the pixel chip is know to be better than $10 \%$.

\section{Measurements of Point Resolution}

We use cosmic ray events to measure the transverse $(x, y)$ point resolution of the detector with $\mathrm{HeCO}_{2}$ gas, as a function of the drift length, $z$. For this study we operate the detector with high gain $\left(>40 \times 10^{3}\right)$ and increased drift field $(1180 \mathrm{~V} / \mathrm{cm})$ for an expected drift velocity of $22.8 \mu \mathrm{m} / \mathrm{ns}$ [33, 34]. Since the chip is sampling charge at a fixed $40 \mathrm{MHz}$, the increased drift velocity degrades the $z$-resolution, but allows 16 pixel chip time bins, the maximum number that can be recorded consecutively, to cover the entire drift gap. This enables an important analysis technique: we select tracks that traverse the entire drift gap and which have hits near the top and bottom of this gap, as shown in Fig. 10. Even though this detector does not otherwise measure absolute $z$, for such events we can assign an absolute $z$ position to each recorded hit. For each event, we fit a straight line to the distribution of hits and determine the $x$ and $y$ point resolution from the distribution of the $x$-distance and $y$-distance between the hit positions and points of closest approach to the line. Though these distances are not generally equal to the true miss-measurements, the two quantities become identical for vertical tracks. We keep tracks within 25 degrees of vertical (z). A GEANT4[35] Monte Carlo simulation estimates that for such tracks our procedure measures the actual $x$ and $y$ point resolutions to an accuracy better than 5 percent. We perform this procedure for each of the $16 z$-coordinates separately, and obtain the transverse point resolution versus drift distance $z$, shown in Fig. 11. We expect the point resolution to be the quadrature sum of the readout plane's point resolution (which is independent of $z$ ), and the transverse diffusion (which is proportional to $\sqrt{z}$ ), i.e.

$$
\sigma_{x / y}^{T}(z)=\sqrt{\left(\sigma_{x / y}^{R}\right)^{2}+C_{T}^{2} z}
$$

where $\sigma_{x / y}^{R}$ is the readout plane point resolution in $x$ or $y, C_{T}$ is the transverse diffusion per $\sqrt{z}$, and $z$ is the drift distance. By fitting this function to the experimental data, we obtain $\sigma_{x}^{R}=(197 \pm 11) \mu m$ and $\sigma_{y}^{R}=(142 \pm 9) \mu m$, in good agreement with the analytical estimates $\sigma_{x}^{R}=184 \mu \mathrm{m}$ and $\sigma_{y}^{R}=143 \mu \mathrm{m}$. Table 3 gives a breakdown of the analytical estimate. The resolution in $y$ is better than that in $x$ because the rectangular pixels are smaller in the $y$ direction $(50 \mu \mathrm{m})$ than the $x$-direction $(400 \mu m)$. Note that in $y$, the readout resolution is not limited 


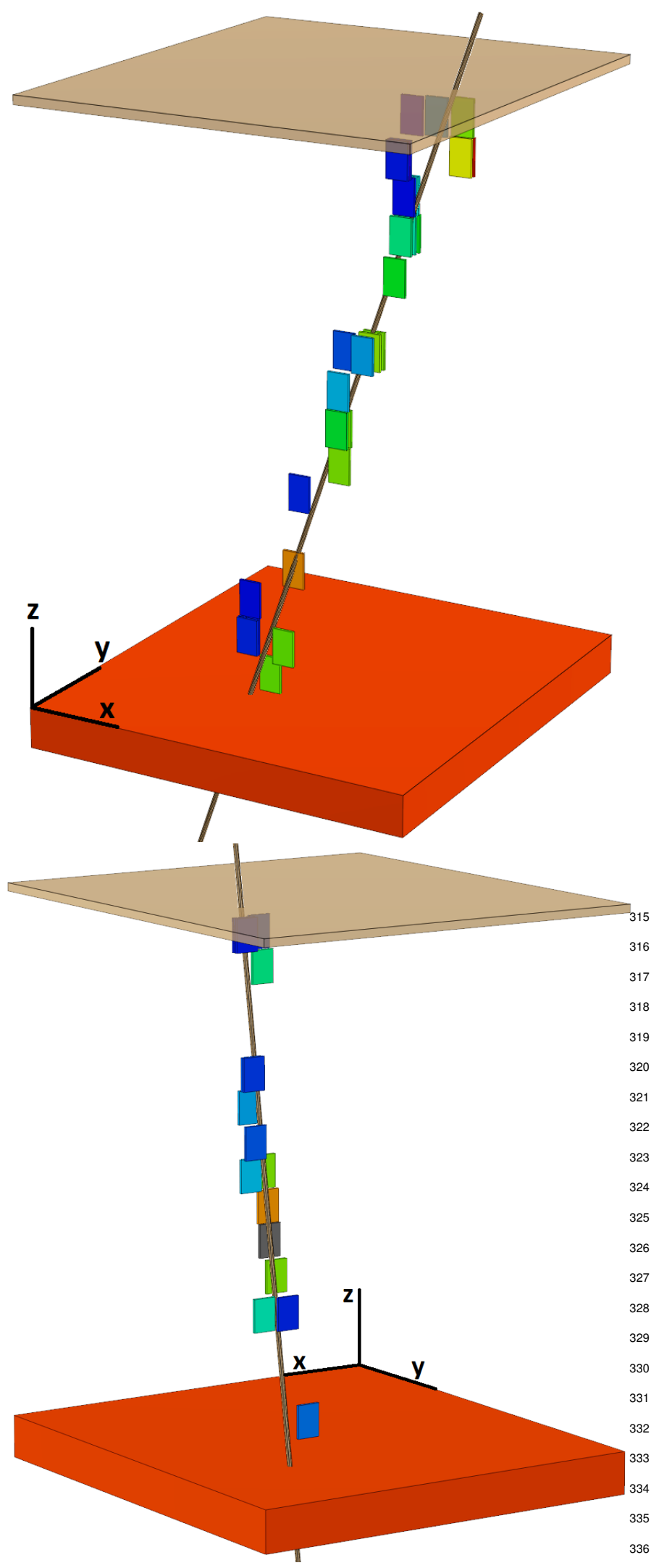

Figure 10: Cosmic ray events measured in $\mathrm{ArCO}_{2}$ gas (top) and $\mathrm{HeCO}_{2}$ gas (bottom) with a detector gain of $4 \times 10^{4}$. The small boxes represent hits recorded by the pixel chip, and measure $50 \mu \mathrm{m} \times 400 \mu \mathrm{m}$ in $x \times y$. Their color (available ${ }^{337}$ online) is determined by the measured ToT, which reflects the ionization density. The line is the best fit to the hits. The volume below the hits symbolizes ${ }^{338}$ the part of the readout plane that projects onto the pixel chip. The top of this 339 volume is the top of GEM1. The transparent volume above the hits is part of the cathode mesh. Assuming these tracks were created by minimum ionizing 8 particles, the total energies deposited are of order $2 \mathrm{keV}\left(\mathrm{ArCO}_{2}\right)$ and $1.0 \mathrm{keV}$ $\left(\mathrm{HeCO}_{2}\right)$, demonstrating the excellent detector sensitivity at high gain settings.
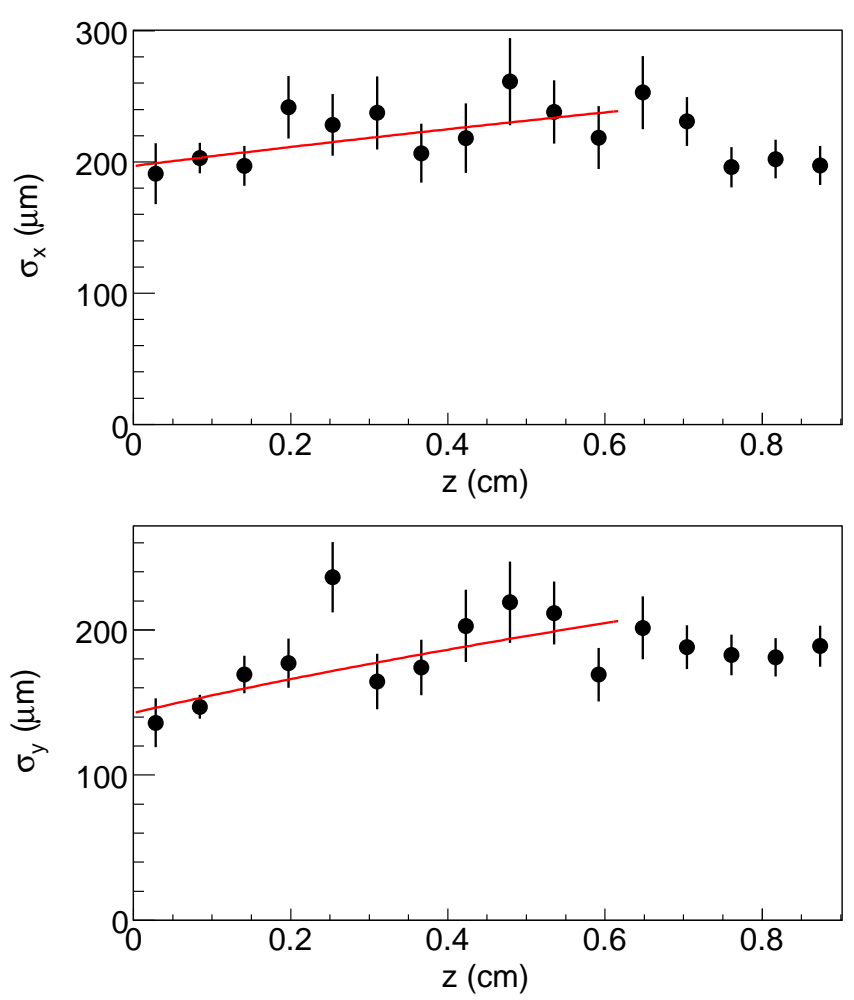

Figure 11: Measured transverse point-resolution in $x$ (upper) and $y$ (lower) versus drift length $(z)$ for cosmic ray tracks in $\mathrm{HeCO}_{2}$ gas. The black points show experimental data, the curves (red in online version) are fits of equation 4 to the data.

by the feature size of either the GEMs or pixels, but by the diffusion in the collection and transfer gaps. As for the diffusion in the drift gap, the fits to $\sigma_{x}(z)$ and $\sigma_{y}(z)$ yield $C_{T}=(172 \pm 46) \mu m$ and $C_{T}=(189 \pm 27) \mu m$, respectively, where the quoted uncertainties are statistical only. The two values are consistent with each other, as expected, but significantly larger than the Magboltz prediction of $C_{T}=109 \mu \mathrm{m}$. The discrepancy may be due to non-uniformities in the drift field, or possibly from impurities in the gas. However, the GEANT4 simulation also suggests that while our analysis procedure is reliable for measuring the point resolution, it adds a systematic uncertainty to the measured diffusion: this is because equation 4 does not account for detector inefficiencies in the case of highly diffuse charge at the edge of the ionization cloud, which can be seen to bias the measured diffusion in Fig. 11 for $z$ greater than $0.6 \mathrm{~cm}$. Hence we exclude this $z$-range from the fit. However, the exact diffusion value measured is very sensitive to the exact $z$-range used, resulting in a systematic uncertainty of order $50 \%$. If accurate diffusion measurements are desired in the future, this may be accomplished by operating the detector at settings where the single electron efficiency approaches unity, so that the fraction of undetected charge becomes negligible.

\section{3-D Tracking of Helium Nuclei: Angular Resolution}

One incidental benefit of using ${ }^{4} \mathrm{He}$ nuclei as our neutron target is that we can use alpha particle sources to estimate the de- 


\begin{tabular}{|c|c|c|}
\hline & $\sigma_{x}(\mu m)$ & $\sigma_{y}(\mu m)$ \\
\hline GEM1 hole spacing & 40.4 & 40.4 \\
\hline transverse diffusion in collection gap & 93.2 & 93.2 \\
\hline GEM2 hole spacing & 40.4 & 40.4 \\
\hline transverse diffusion in transfer gap & 91.8 & 91.8 \\
\hline pixel size & 115 & 14.4 \\
\hline Predicted $\sigma_{x, y}$ & 184 & 143 \\
\hline Measured $\sigma_{x, y}$ & $197 \pm 11$ & $142 \pm 9$ \\
\hline
\end{tabular}

Table 3: Estimated contributions to the readout plane resolution. The collection ${ }^{352}$ gap, transfer gap, and coordinate system are defined in Fig. 3. Diffusion values ${ }^{353}$ were calculated with Magboltz [33] and the GEM and pixel resolution values 354 are the respective feature sizes divided by $\sqrt{12}$. The predicted resolution is the quadrature-sum of the contributions.

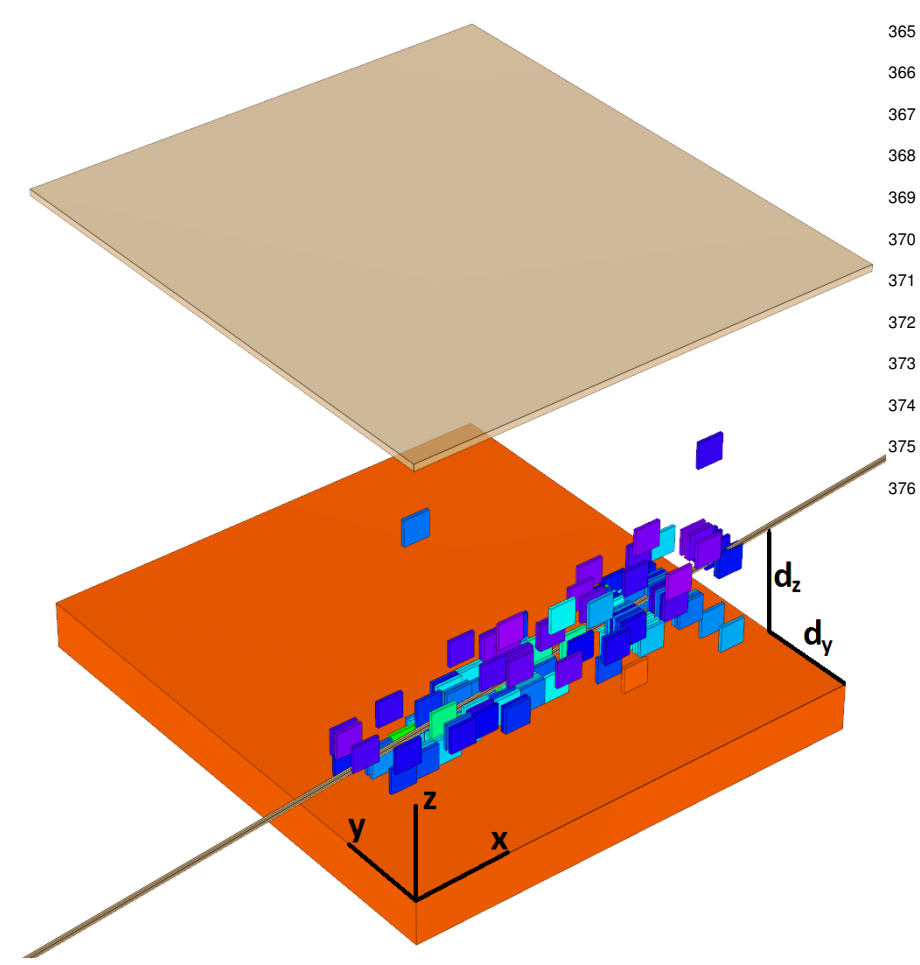

Figure 12: Alpha particle track segment measured in $\mathrm{HeCO}_{2}$ gas with a detector377 gain of $3 \times 10^{3}$. The Po-210 alpha source is located beyond the right edge of ${ }_{378}$ the image, at $x \approx 46 \mathrm{~mm}$ Because the detector does not measure absolute $z$, however, we always display events with the lowest hit at the bottom of the drift $^{379}$ gap, and so the track appear lower than the actual source position. tector performance for detecting He-recoils. We estimate the angular resolution with two complementary methods, described in the next two paragraphs. Both methods make use of the same data sample, recorded with a Po-210 (5.3 MeV) alpha source located at $x \approx 46 \mathrm{~mm}$. When recording this particular data set, the two outer columns of the pixel chip were disabled, due to individual noisy pixels in those columns. The source is oriented so that the emitted alpha particles traverse the drift gap at $z \approx 5 \mathrm{~mm}$, at an angle $\theta \approx 80 \mathrm{deg}$. Due to the large ionization density in these events, we operate the detector with a reduced gain of 3200. We select a pure sample of well-reconstructed alpha tracks by fitting the events with a straight line and requiring $L>5000 \mu \mathrm{m}, \chi^{2} / n . d . f$. $<2.0$, and $N>60$, where $L$ is the track length, $\chi^{2} / n . d . f$. is the reduced chi-squared, and $N$ is the number of pixels hit. To avoid cases were tracks graze the sensitive volume and not all hits are detected, we veto events with hits within $400 \mu \mathrm{m}$ of the $y$-edges of the chip. Figure 12 shows an example of an event passing the selection.

Method I estimates the angular resolution from the event-byevent uncertainties reported by the track-fitter. This requires that the $\chi^{2}$ used to fit the tracks is calculated with the correct point resolutions. We use the residual distributions from the alpha track sample to obtain $\sigma_{y}=(275 \pm 0.7) \mu \mathrm{m}$, and $\sigma_{z}=(313 \pm 62) \mu \mathrm{m}$. Because most alpha tracks are nearly parallel to the $x$-axis, these events don't really constrain $\sigma_{x}$. Since, except for the pixel size, our detector is symmetric in $x$ and $y$, we instead obtain $\sigma_{x}=(298 \pm 1) \mu \mathrm{m}$ by subtracting the pixel $\mathrm{y}-$ resolution (Table 3 ) in quadrature from $\sigma_{y}$, and then adding the pixel x-resolution in quadrature. Our final results are not sensitive to the exact choice of $\sigma_{x}$. Note that these point resolutions for alpha particles are slightly larger than the point resolutions measured for cosmic ray events (Fig. 11) at $z=0.5 \mathrm{~cm}$ (the approximate vertical position of the alpha tracks). This, presumably, is mainly due to straggling. The track angle resolutions reported by the track fitter are shown in Fig. 13. Fitting for the mean resolution with a Gaussian gives $\sigma_{\phi}=(1.068 \pm 0.003)^{\circ}$ and $\sigma_{\theta}=(1.202 \pm 0.004)^{\circ}$.

Method II extracts the angular resolution for alpha tracks analytically. This allows the generalization of our results to a wider set of track types. Assuming the charge is deposited uniformly along a straight line (according to a SRIM simulation [36], this is a good approximation for the short mid-section of the alpha-particle trajectories measured), it can be shown by a simple estimate that the track angle resolution depends on the point resolution as

$$
\sigma_{\phi, \theta}=\frac{\sqrt{12} \sigma_{y, z}}{L \sqrt{N}} \text { (radians) }
$$

Here, $\sigma_{\phi, \theta}$ is the point resolution in the coordinate direction that determines the angle of interest, $L$ is the track length, and $N$ is the number of space-points measured. The alpha tracks considered here traverse the whole chip width in $x$, but two columns are disabled, so that $L \approx 6.4 \mathrm{~mm}$. Because the tracks are roughly parallel to the $x$-axis, we use $\sigma_{y}=275 \mu \mathrm{m}$ when calculating $\sigma_{\phi}$ and $\sigma_{z}=313 \mu \mathrm{m}$ when calculating $\sigma_{\theta}$. In order to verify the predicted dependence of the resolution on $N$ 

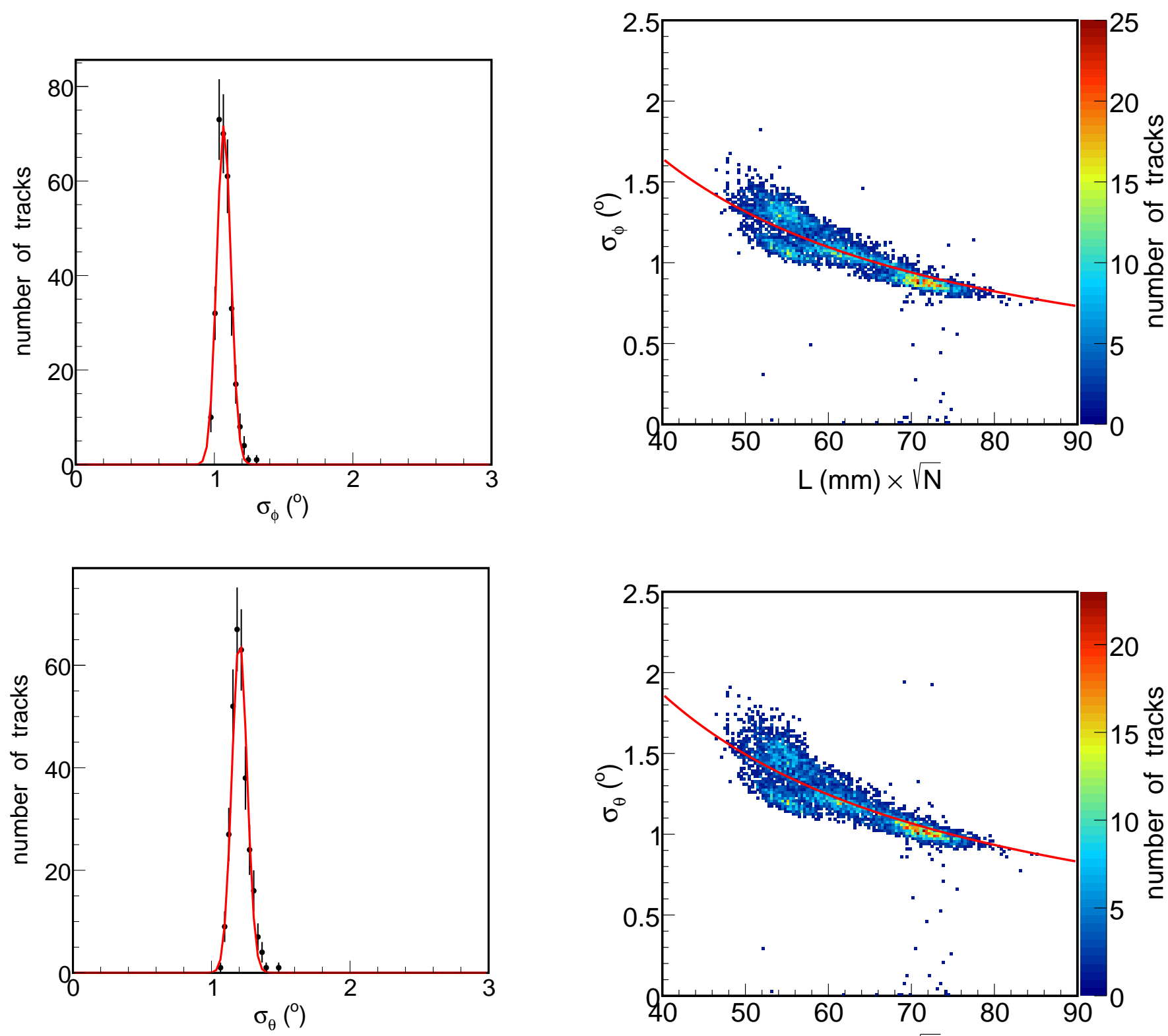

Figure 13: Track fitter uncertainties (black data points) in azimuthal angle $(\phi)$ (top) and polar angle $(\theta)$ (bottom), for alpha particle events measured in $\mathrm{HeCO}_{2}$ gas. The curves show Gaussian fits to the data.

Figure 14: Track fitter uncertainties in $\phi$ (top) and $\theta$ (bottom) versus track length $(L)$ and number of pixels hit $(N)$ for alpha-particle events. The smooth curves are fits of Equation 5 to the distributions. 


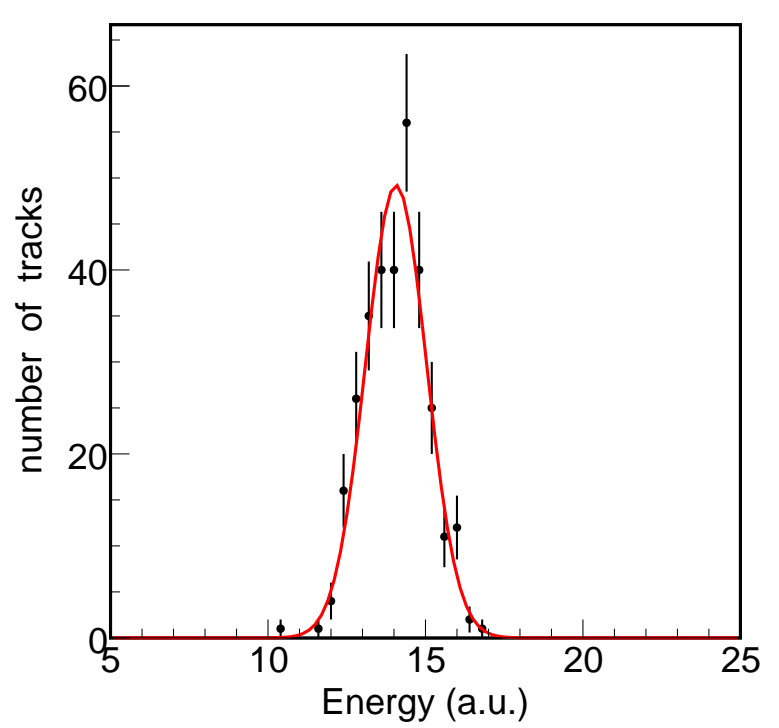

Figure 15: Reconstructed (uncalibrated) energy of alpha-particle events, ${ }^{434}$ recorded with a Po-210 source placed $3.88 \mathrm{~cm}$ from the detector, which was ${ }^{435}$ operating with $\mathrm{HeCO}_{2}$ gas. The black points show experimental data, and the 436 curve is a Gaussian fit. and $L$, we remove our selection on the impact parameter. This ${ }^{439}$ yields a set of tracks with a larger variation in the number of ${ }^{440}$ spacepoints, because the tracks traverse regions of the chip with ${ }^{441}$ different efficiencies. Figure 14 shows the resolution reported ${ }^{442}$ by the track fitter for these events. The red line in the figure ${ }^{443}$ shows the result of fitting Equation 5 to the data points, where ${ }^{444}$ we allow $\sigma_{y, z}$ to float in the fit. The analytical expression ap- ${ }^{445}$ pears to account correctly for the dependence of the resolution on track length and the number of space points measured. The point resolutions obtained from the fit are $\sigma_{y}=(295 \pm 1) \mu \mathrm{m}^{446}$ and $\sigma_{z}=(334 \pm 1) \mu \mathrm{m}$, both $7 \%$ higher than the actual point resolutions. While not a precise agreement, this means that the ${ }^{447}$ track angle resolution for a range of track lengths and num- ${ }^{448}$ ber of measurement points can be predicted at the $10 \%$-level ${ }^{449}$ from the point resolution, which in turn can be predicted reli- ${ }^{450}$ ably from analytical estimates based on pixel and GEM feature ${ }^{451}$ sizes, as demonstrated in Section 5. The present work thus pro- ${ }^{452}$ vides a validated foundation for optimizing future, larger $\mathrm{de}_{-}{ }^{453}$ tectors, and for estimating the performance requirements on the $\mathrm{e}^{454}$ components needed.

\section{3-D Tracking of Helium Nuclei: Energy Resolution}

We estimate the fractional energy resolution at approxi-460 mately $550 \mathrm{keV}$ by using the alpha particle sample discussed 461 in the previous section. The event selection remains the same,462 including the impact parameter cut used for method I. The ion-463 ization energy corresponding to each reconstructed track is ob-464 tained by converting the time over threshold (ToT) measured ${ }_{465}$ by each pixel into an energy value, and adding up the energy 466 values for all pixels in the event. The conversion from ToT to 467 energy requires a calibration of the ToT measurement in each ${ }_{468}$ pixel, which is performed with a charge injection circuit inter-469 nal to the pixel chip. The calibration requires knowledge of the gain, which was measured as described in Section 4, and the work function $W$ for the gas. After performing this calibration procedure, the energy scale of the detector should be uniform across pixels. Because of the non-uniform charge collection discussed in Section 3, however, it is not, and instead varies strongly across the chip, with a different response in each 400$\mu \mathrm{m}$ pixel column. Studies at LBNL [37] revealed that this is caused by imperfect contact between the chip and the conductive layer that was deposited onto it, so that at certain positions some of the GEM avalanche charge does not reach the pixel chip. We have already built an upgraded detector with the next generation (FE-I4) pixel chip, where we employed improved metal deposition, and this seems to have resolved the issue [38]. Once we realized that the metal layer was the culprit, we gave up on an absolute energy scale calibration of the current detector, and we quote energy measurements here in uncalibrated, arbitrary units (a.u.). Figure 15 shows the energy measured with the pixel chip for the same alpha sample as was used to measure the angular resolution. A Gaussian fit determines the fractional energy resolution to be $\sigma_{E} / E=6.9 \%$. The energy resolution is good, but quite a bit worse than the outstanding gain resolution, expected to be of order $2-3 \%$ for $550 \mathrm{keV}$ signals and the gain used. The discrepancy appears to be due to the imperfect metallization. (We also point out that the measured resolution would be even worse for tracks at a different azimuthal angle $\phi$, which may be more sensitive to the particular column-dependent performance observed here.) Also the energy resolution is improved in our next-generation detector [38].

\section{Directional Detection of Fast Neutrons}

Having established the performance of the $\mathrm{D}^{3}$-Micro prototype, we expose it to a $50 \mu \mathrm{Ci} \mathrm{Cf}-252$ fast neutron source. The average expected track length of reconstructed He-recoils from neutron-He elastic scattering is $1.6 \mathrm{~mm}$ at atmospheric pressure, which should be easy to detect, given the established detector performance. However, given the tiny detector volume and hence scattering probability, a very long exposure is required. Figure 16 shows the event rate versus 3-D recoil angle for all events with at least two pixel hits, for a 56-day sourcefree background run and a 7.5-day run with the neutron source $47 \mathrm{~cm}$ from the detector. The event rate is 27-sigma larger with the source present, the excess seen agrees with expectations from simulation [10], and the observed recoil angle distribution points back to the source. Clearly, already this tiny prototype can detect fast neutrons, and locate a point-like source in 3-D. More quantitative detail on the performance of a larger detector will be published soon.

Note that the width of the recoil angle distribution is mainly due to scattering kinematics; the contribution from detector resolution (Section 6) is small. Figure 17 shows one of the neutron-recoil event candidates measured with the Cf-252 source present. The measured track is $5.0 \mathrm{~mm}$ long. Based on SRIM, this corresponds to recoil and ionization energies of 

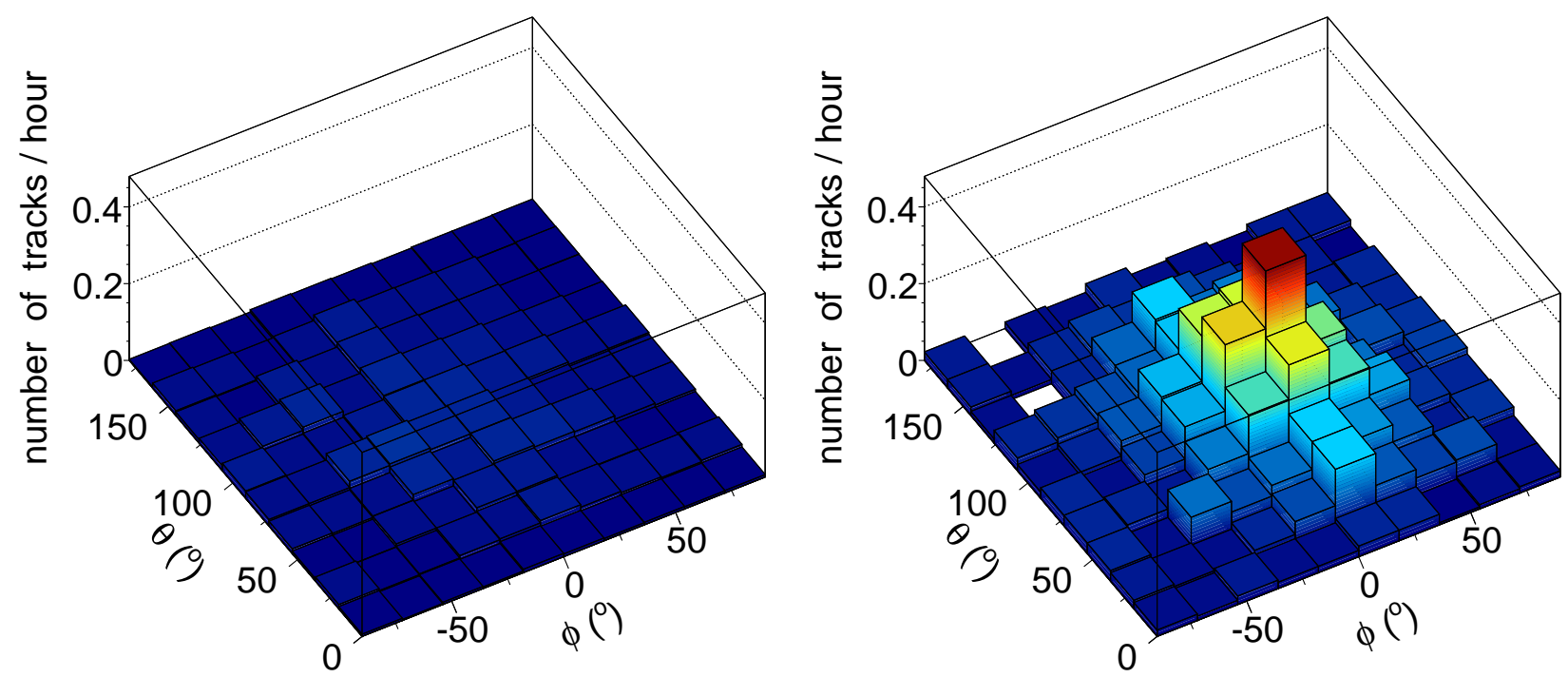

Figure 16: Time-normalized recoil angle distribution without (left) and with (right) a Cf-252 fast neutron source near the detector. The source is detected with high significance, and the recoils point back to the source.

$350 \pm 50 \mathrm{keV}$, where the quoted uncertainy is an estimate of field non-uniformity and diffusion effects.

The seemingly minor mechanical modifications we made to the support structure for this particular study (Fig. 2) were crucial for obtaining the clean directional signal. We simulated a range of drift gap configuration in COMSOL, and chose the largest gap were the effect of drift field distortions were deemed acceptable. This increased the signal efficiency by a factor of five. We also reduced the amount of plastic in the support structure, which lowered the rate of the two main backgrounds, protons from neutron scattering in the plastic and alpha-particles not related to the neutron source (presumably from decaying radon-progeny stuck to the plastic) by an order of magnitude.

\section{Discussion of Results and Conclusion}

We demonstrated the 3-D reconstruction of mm-length track segments from cosmics, alpha particles, and nuclear recoils. We investigated two main performance measures of interest in that context, angular resolution and energy resolution, and how these are determined by choice of detector components, geometry, and gas mixture.

The point (single-hit) resolution of the detector can be reliably predicted from the GEM hole spacing, pixel segmentation, and diffusion in the different detector regions, and was found to be of order $200 \mu m \oplus C \sqrt{z}$, where the first term is the resolution of the readout plane, $C$ is the diffusion per $\sqrt{z}, z$ is the drift length, and $\oplus$ denotes addition in quadrature. Interestingly, in the most precise coordinate, $y$, the resolution of the readout plane is no longer limited by detector segmentation, but rather diffusion in the transfer and collection gaps. We showed how the point resolution in turn determines the track angle resolution, and how this resolution scales with track parameters. For

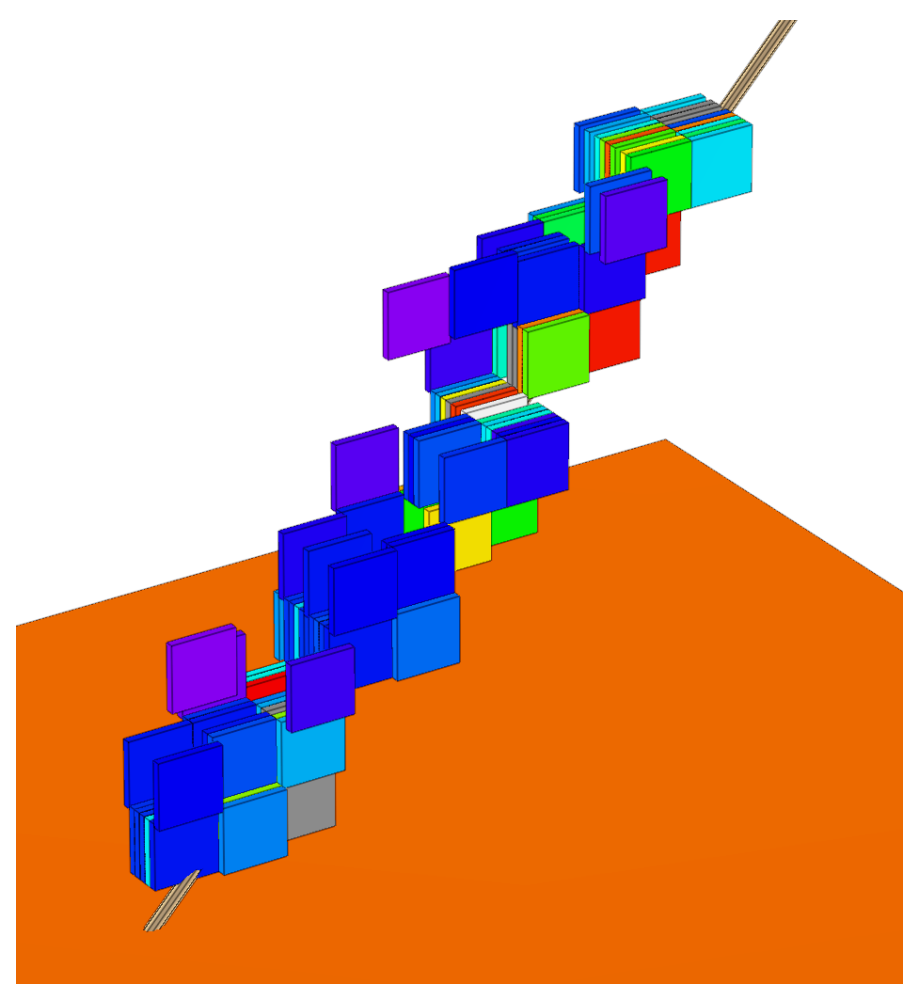

Figure 17: He-recoil candidate in $\mathrm{HeCO}_{2}$ gas, measured with a $\mathrm{Cf}-252$ source near the detector. The measured track is $5.0 \mathrm{~mm}$ long, corresponding to a recoil energy and ionization energy of approximately $350 \mathrm{keV}$. 
the mm-length alpha-track segments studied, the angular reso-556 lution was of order 1 degree. By combining equations 4 and 5 , we find that the angular resolution in larger detectors will be ap-557 proximately $\sqrt{12}(200 \mu m \oplus C \sqrt{z}) /(L \sqrt{N})$, where $L$ is the track558 length, $N$ is the number of space points, and $C$ is the diffusions59 per $\sqrt{z}$. The implication is that tracks of length $5-10 \mathrm{~mm}$ can be 560 reconstructed with angular resolution of order a few degrees in 561 detectors with electron $\operatorname{drift}(C \approx 200 \mu \mathrm{m} / \sqrt{\mathrm{cm}})$ and short drift562 length $(z \lesssim 10 \mathrm{~cm})$. For detectors with longer drift length or im-563 proved track angle resolution, negative ion drift $[39,40]$ would 564 be advantageous to reduce the otherwise dominant contribution from diffusion in the drift gap.

The energy resolution measured for $\mathrm{MeV}$-scale signals was ${ }_{560}$ $\sigma_{E} / E=7 \%$, limited by a position dependence in the charge ${ }_{567}$ collection efficiency due to imperfect contact between the pixel ${ }^{568}$ chip and a metal layer that was deposited onto it. This has ${ }_{570}^{569}$ been resolved in the next generation detector [38]. The mea ${ }_{571}^{570}$ sured asymptotic (high-gain) gain resolution, which we expect572 to limit the energy resolution in future detectors, varies from ${ }^{573}$ $\sigma_{E} / E=15 \%$ at $3 \mathrm{keV}$, limited by statistical fluctuations in $_{575}^{574}$ the primary ionization and in the gas avalanche process, to $\mathrm{t}_{576}$ $\sigma_{E} / E=2 \%$ at the MeV-scale, limited by detector and mea-577 surement stability. Improved gain resolution and thus energy ${ }^{578}$ resolution at low energies may be achieved in future detectors ${ }^{579}$ by counting electrons individually, which may also require neg- ${ }_{-581}$ ative ion drift [32].

The TPC charge readout technology under study appears ${ }_{585}^{584}$ promising for future directional WIMP and neutron detectors.586 The angular and gain resolutions measured are excellent, and587 the 3-D tracking capability would reduce the number of signal ${ }^{588}$ events needed to claim a dark matter observation. The tech ${ }_{590}^{589}$ nology studied has other suitable attributes, among them neg-591 ligible noise rates and high sensitivity. More precise studies592 are needed, but for now, the lack of noise hits in our event dis- ${ }^{593}$ plays and the successful 3-D reconstruction of cosmic ray in ${ }^{-594}$ duced tracks with energies of order $1 \mathrm{keV}$ suggest good poten-596 tial for achieving directional detection with low energy thresh-597 old. This will be important in the context of searching for the ${ }_{599}^{598}$ $\mathrm{keV}$-scale nuclear recoils expected from the interaction of low-600 mass $(\approx 10 \mathrm{GeV})$ WIMPs. In the work presented we focused 601 on $\mathrm{MeV}$-scale nuclei and atmospheric gas pressure. We did not ${ }^{602}$ operate at low gas pressure $\left(\approx 10-50\right.$ Torr), which is required to ${ }_{604}^{603}$ extend the length of keV-scale recoils to measurable size [10],605 nor did we use target gases optimized for WIMP sensitivity. We606 have already performed other work in this direction [9]. Further ${ }^{607}$ measurements with additional gas mixtures, e.g. $\mathrm{CF}_{4}, \mathrm{CF}_{4}: \mathrm{CS}_{2609}{ }_{608}$ [14] and $\mathrm{CF}_{4}: \mathrm{CS}_{2}: \mathrm{O}_{2}$ [41] at low gas pressures are required and $\mathrm{d}_{610}$ planned. For low-mass WIMP searches, another outstanding611 question is the significance of straggling for $\mathrm{keV}$-scale recoils, ${ }^{612}$ which we plan to measure. As for neutron detection, we demon ${ }_{614}^{613}$ strated that the technology under study can locate a fast neu-615 tron source in 3-D. We expect that the next generation detector, ${ }^{616}$ where the energy measurement has been improved [38], should ${ }^{617}$ be able to simultaneously locate and measure the neutron spec- ${ }_{619}^{618}$ trum of a source.

\section{Acknowledgements}

We thank Marc Rosen for his assistance in designing the support structure and test vessel. We thank Blake Pollard and Kamaluoawaiku Beamer for performing electric field simulations. We thank Peter Lewis for assistance during the revision process. We acknowledge support from the U.S. Department of Homeland Security under Award Number 2011-DN-077-ARI050-03 and the U.S. Department of Energy under Award Number DESC0007852.

\section{References}

[1] D. R. Nygren and J. N. Marx, "The Time Projection Chamber," Phys. Today 31N10, 46 (1978).

[2] R. Bellazzini, F. Angelini, L. Baldini, F. Bitti, A. Brez, L. Latronico, M. M. Massai and M. Minuti et al., "Reading a GEM with a VLSI pixel ASIC used as a direct charge collecting anode," Nucl. Instrum. Meth. A 535, 477 (2004).

[3] P. Colas, A. P. Colijn, A. Fornaini, Y. Giomataris, H. van der Graaf, E. H. M. Heijne, X. Llopart and J. Schmitz et al., "The readout of a GEMor micromegas-equipped TPC by means of the Medipix2 CMOS sensor as direct anode," Nucl. Instrum. Meth. A 535, 506 (2004).

[4] T. Kim, M. Freytsis, J. Button-Shafer, J. Kadyk, S. E. Vahsen and W. A. Wenzel, "Readout of TPC tracking chambers with GEMs and pixel chip," Nucl. Instrum. Meth. A 589, 173 (2008).

[5] T. Thorpe and S. Vahsen, "Unexpected Inefficiencies in TPCs with Pixel Chip Readout", in preparation (2014).

[6] I.S. Seong, K. Beamer, M.T. Hedges, I. Jaegle, M.D. Rosen, S.J. Ross, T.N. Thorpe, S.E. Vahsen, and J. Yamaoka, "Time Projection Chambers with Integrated Pixels and their Application to Fast Neutron Detection and Dark Matter Searches," Nucl. Instrum. Meth. A PROCEEDINGS-D13-00102 (2013).

[7] S. J. Ross, M. T. Hedges, I. Jaegle, M. D. Rosen, I. S. Seong, T. N. Thorpe, S. E. Vahsen and J. Yamaoka, "Charge-focusing readout of time projection chambers," Nuclear Science Symposium and Medical Imaging Conference Record (NSS/MIC), 2012 IEEE, 1760 (2012).

[8] J. Yamaoka, H. Feng, M. Garcia-Sciveres, I. Jaegle, J. Kadyk, Y. Nguyen, M. Rosen, S. Ross, T. Thorpe, S. Vahsen, "Application of Time Projection Chambers with GEMs and Pixels to WIMP Searches and Fast Neutron Detection," Physics Procedia, 37, 567 (2012).

[9] S. Vahsen, K. Oliver-Mallory, M. Lopez-Thibodeaux, J. Kadyk and M. Garcia-Sciveres, "Tests of gases in a mini-TPC with pixel chip readout," Nucl. Instrum. Meth. A 738, 111 (2014).

[10] I. Jaegle, H. Feng, S. Ross, J. Yamaoka and S. E. Vahsen, "Simulation of the Directional Dark Matter Detector $\left(D^{3}\right)$ and Directional Neutron Observer (DiNO)," EAS Publ. Ser. 53, 111 (2012).

[11] D. N. Spergel, "The Motion of the Earth and the Detection of Wimps," Phys. Rev. D 37, 1353 (1988).

[12] P. Grothaus, M. Fairbairn and J. Monroe, "Directional Dark Matter Detection Beyond the Neutrino Bound," arXiv:1406.5047 [hep-ph].

[13] S. Ahlen, J. B. R. Battat, T. Caldwell, C. Deaconu, D. Dujmic, W. Fedus, P. Fisher and F. Golub et al., "First Dark Matter Search Results from a Surface Run of the 10-L DMTPC Directional Dark Matter Detector," Phys. Lett. B 695, 124 (2011)

[14] E. Daw, J. R. Fox, J. L. Gauvreau, C. Ghag, L. J. Harmon, M. Gold, E. R. Lee and D. Loomba et al., "Spin-Dependent Limits from the DRIFTIId Directional Dark Matter Detector," Astropart. Phys. 35, 397 (2012)

[15] S. E. Vahsen, H. Feng, M. Garcia-Sciveres, I. Jaegle, J. Kadyk, Y. Nguyen, M. Rosen and S. Ross et al., "The Directional Dark Matter Detector $\left(D^{3}\right)$," EAS Publ. Ser. 53, 43 (2012)

[16] D. Santos, J. Billard, G. Bosson, J. L. Bouly, O. Bourrion, C. .Fourel, O. Guillaudin and F. Mayet et al., "MIMAC : A micro-tpc matrix for directional detection of dark matter," EAS Publ. Ser. 53, 25 (2012)

[17] K. Miuchi, H. Nishimura, K. Hattori, N. Higashi, C. Ida, S. Iwaki, S. Kabuki and H. Kubo et al., "First underground results with NEWAGE0.3 a direction-sensitive dark matter detector," Phys. Lett. B 686, 11 (2010) 
[18] T. Naka, M. Kimura, M. Nakamura, O. Sato, T. Nakano, T. Asada, Y. Tawara and Y. Suzuki, "R\&D Status of Nuclear Emulsion For Directional Dark Matter Search," EAS Publ. Ser. 53, 51 (2012)

[19] A. Drukier, K. Freese, D. Spergel, C. Cantor, G. Church and T. Sano, "New Dark Matter Detectors using DNA for Nanometer Tracking," arXiv:1206.6809 [astro-ph.IM].

[20] D. R. Nygren, "Columnar recombination: a tool for nuclear recoil directional sensitivity in a xenon-based direct detection WIMP search," J. Phys. Conf. Ser. 460, 012006 (2013).

[21] S. Ahlen et al., "The case for a directional dark matter detector and the status of current experimental efforts," Int. J. Mod. Phys. A 25, 1 (2010).

[22] A. M. Green and B. Morgan, "Optimizing WIMP directional detectors," Astropart. Phys. 27, 142 (2007).

[23] F. Sauli, "GEM: A new concept for electron amplification in gas detectors," Nucl. Instrum. Meth. A386, 531-534 (1997).

[24] G. Aad, M. Ackers, F. A. Alberti, M. Aleppo, G. Alimonti, J. Alonso, E. C. Anderssen, A. Andreani et al., "ATLAS pixel detector electronics and sensors," JINST 3, P07007 (2008).

[25] S. Bachmann, A. Bressan, L. Ropelewski, F. Sauli, A. Sharma and D. Mormann, "Charge amplification and transfer processes in the gas electron multiplier," Nucl. Instrum. Meth. A 438, 376 (1999).

[26] M. Oreglia, "A Study of the Reactions $\psi^{\prime} \rightarrow \gamma \gamma \psi$," SLAC Stanford SLAC-236, 226 (1980).

[27] A. Sharma, "Properties of some gas mixtures used in tracking detectors," SLAC-J-ICFA-16-3, 19 (1998).

[28] G. F. Knoll, "Radiation Detection and Measurement," New York, USA: John Wiley \& Sons (2000).

[29] U. Fano, "Ionization Yield of Radiations. 2. The Fluctuations of the Number of Ions," Phys. Rev. 72, 26 (1947)

[30] G.D. Alkhazov, "Statistics of electron avalanches and ultimate resolution of proportional counters," Nucl. Instrum. Meth. 89, 155 (1970).

[31] Akira Hashiba, Kimiaki Masuda, Tadayoshi Doke, Tan Takahashi, Yuzo Fujita, "Fano factor in gaseous argon measured by the proportional scintillation method," Nucl. Instrum. Meth. A 227, 305 (1984).

[32] P. Sorensen, M. Heffner, A. Bernstein, J. Renner and M. Sweany, "Towards energy resolution at the statistical limit from a negative ion time projection chamber," Nucl. Instrum. Meth. A 686, 106 (2012)

[33] All drift velocities used were calculated with the CERN-produced software Magboltz, version 10.0.1, 10. http://consult.cern.ch/writeup/magboltz/

[34] S. F. Biagi, "Monte Carlo simulation of electron drift and diffusion in counting gases under the influence of electric and magnetic fields," Nucl. Instrum. Meth. A 421, no. 1-2, 234 (1999).

[35] S. Agostinelli et al. [GEANT4 Collaboration], "GEANT4: A Simulation toolkit,” Nucl. Instrum. Meth. A 506, 250 (2003).

[36] J.F. Ziegler, M.D. Ziegler, J.P. Biersack, "SRIM The stopping and range of ions in matter," Nucl. Instrum. Meth. B 268, 1818-1823 (2010).

[37] J. Kadyk, personal communication (2014).

[38] I. Jaegle, S.E. Vahsen, et al., "High Resolution 3-D Tracking in a TPC with Pixel Readout", in preparation (2014).

[39] C. J. Martoff, D. P. Snowden-Ifft, T. Ohnuki, N. Spooner and M. Lehner, "Suppressing drift chamber diffusion without magnetic field," Nucl. Instrum. Meth. A 440, 355 (2000).

[40] J. Miyamoto, I. Shipsey, C. J. Martoff, M. Katz-Hyman, R. Ayad, G. Bonvicini and A. Schreiner, "GEM operation in negative ion drift gas mixtures,” Nucl. Instrum. Meth. A 526, 409 (2004).

[41] D. P. Snowden-Ifft, "Discovery of multiple, ionization-created CS2 anions and a new mode of operation for drift chambers," Rev. Sci. Instrum. 85, 013303 (2014). 